\title{
Quantum limits on the time-bandwidth product of an optical resonator
}

\author{
MANKEI TSANG ${ }^{1,2, *}$ \\ ${ }^{1}$ Department of Electrical and Computer Engineering, National University of Singapore, 4 Engineering Drive 3, Singapore 117583 \\ ${ }^{2}$ Department of Physics, National University of Singapore, 2 Science Drive 3, Singapore 117551 \\ mankei@nus.edu.sg
}

Compiled December 25, 2017

\begin{abstract}
A thought-provoking proposal by Tsakmakidis et al. [Science 356, 1260 (2017)] suggests that nonreciprocal optics can break a time-bandwidth limit to passive resonators. Here I quantize their resonator model and show that quantum mechanics does impose a limit, or else requires extra noise to be added in the same fashion as amplified spontaneous emission in an active resonator. I also use thermodynamics to argue that extra dissipation or noise must be present in their proposed device. (c) 2017 Optical Society of America
\end{abstract}

OCIS codes: (270.2500) Fluctuations, relaxations, and noise; (230.5750) Resonators

\section{http://dx.doi.org/10.1364/ao.XX.XXXXXX}

Nonreciprocal optics has enjoyed a renaissance in recent years [1-8]. While the main driver has been the practical desire to make on-chip isolators and circulators for integrated photonics, a recent proposal by Tsakmakidis et al. [9] suggests that nonreciprocal optics can break a fundamental time-bandwidth limit to passive resonators. If true, the proposal promises an upheaval of fundamental physics, as well as new functionalities such as broadband slow light. Their models and simulations are based exclusively on classical electromagnetism, however, leaving open the question whether they may violate other fundamental laws of physics, especially quantum mechanics and thermodynamics.

In this Letter, I quantize their resonator model using textbook quantum optics [10-12] and show that, unlike classical electromagnetism, quantum mechanics does impose a timebandwidth limit to passive resonators. To break the limit, the quantum theory requires extra noise to be added in the same fashion as amplified spontaneous emission in an active resonator. Going deeper into the details of their proposed implementation, I also find that it requires a one-way power-transfer mechanism that violates the second law of thermodynamics. These findings suggest that their proposal is unlikely to be physical, or can be accomplished by a conventional active resonator if the extra noise is acceptable.

To set the stage, I first recall basic facts regarding passive linear optics and nonreciprocity in quantum optics. The quantum Hamiltonian for any multi-mode passive linear optics has the general form

$$
H=\sum_{j} b_{j}^{\dagger} G_{j k} b_{k}
$$

where $\left\{b_{j}\right\}$ is a set of annihilation operators for the optical modes, + denotes the Hermitian conjugate, and $G$ is a modecoupling matrix [13]. Since $H$ must be an Hermitian operator, $G$ must an Hermitian matrix $\left(G_{j k}=G_{k j}^{*}\right)$. If I identify the $j=0$ mode as the resonator mode with $b_{0}=a$ and the rest as reservoir modes, the Hamiltonian becomes $H=G_{00} a^{\dagger} a+$ $\sum_{k \neq 0}\left(G_{0 k} a^{\dagger} b_{k}+G_{0 k}^{*} a b_{k}^{\dagger}\right)+\sum_{j \neq 0, k \neq 0} b_{j}^{\dagger} G_{j k} b_{k}$. Denote the submatrix $G_{j k}$ for $j \neq 0$ and $k \neq 0$ by $G^{\prime}$. It is also Hermitian, so it can be diagonalized as $G^{\prime}=V^{\dagger} D V$, where $V$ is unitary, $D$ is diagonal and real, and + also denotes the conjugate transpose of a matrix. Defining the reservoir eigenmodes via $c_{j}=\sum_{k \neq 0} V_{j k} b_{k}$ leads to the Hamiltonian

$$
H=G_{00} a^{\dagger} a+\sum_{j}\left(\eta_{j} a^{\dagger} c_{j}+\eta_{j}^{*} a c_{j}^{\dagger}\right)+\sum_{j} D_{j j} c_{j}^{\dagger} c_{j},
$$

where $\eta_{j} \equiv \sum_{k \neq 0} G_{0 k} V_{j k}^{*}$. This Hamiltonian is the starting point for deriving the quantum Langevin equation for a lossy resonator in the Markovian limit [10-12].

Nonreciprocity in classical optics is commonly regarded as an analogy of time-reversal-symmetry breaking in quantum mechanics [1-4]. To go beyond mere analogy and define timereversal symmetry rigorously in quantum optics, consider the proposal by Koch et al. [5]. Let the anti-unitary time-reversal operator be $\Theta$. Time-reversal symmetry in general is defined by the condition $\Theta H \Theta^{-1}=H$ [14]. If $\left\{b_{j}\right\}$ corresponds to a set of spatially localized modes, Koch et al.'s definition of $\Theta$ leads to $\Theta b_{j}^{\dagger} \Theta^{-1}=\exp \left(i \theta_{j}\right) b_{j}^{\dagger}$ and $\Theta b_{j} \Theta^{-1}=\exp \left(-i \theta_{j}\right) b_{j}$, where $\theta_{j}$ is a phase that represents a choice of gauge. Imposing the symmetry on Eq. (1),

$$
\Theta H \Theta^{-1}=\sum_{j} b_{j}^{\dagger} \exp \left(i \theta_{j}-i \theta_{k}\right) G_{j k}^{*} b_{k}=\sum_{j} b_{j}^{\dagger} G_{j k} b_{k},
$$

which implies

$$
\exp \left(i \theta_{j}-i \theta_{k}\right) G_{j k}^{*}=G_{j k} .
$$

A $G$ matrix that cannot satisfy Eq. (4) for any $\left\{\theta_{j}\right\}$ breaks the time-reversal symmetry; the optical circulator is an example [5]. 
For the purpose of this Letter, Eq. (4) is simply an additional constraint on $G$ and irrelevant to the validity of Eq. (2), so I can proceed with the standard formalism of quantum Langevin equations that originate from Eq. (2).

The simplest quantum Langevin equation for a lossy resonator is given by [10-12]

$$
\frac{d a(t)}{d t}=-i \omega_{0} a(t)-\frac{1}{\tau} a(t)+\sqrt{\gamma_{0}} A_{0}(t),
$$

where $a(t)$ is the annihilation operator of the resonator mode in the Heisenberg picture, $A_{0}(t)$ is the annihilation operator of a field at the input of the resonator, $\omega_{0}$ is the resonance frequency, $\tau$ is the decay time of the resonator mode, and $\gamma_{0} \geq 0$ is the input coupling rate. Taking the expectation of Eq. (5) and substituting $\alpha(t)=\sqrt{\gamma_{0} \hbar \Omega}\left\langle a^{\dagger}(t)\right\rangle$ and $s_{\text {in }}(t)=\sqrt{\hbar \Omega}\left\langle A_{0}^{\dagger}(t)\right\rangle$, where $\Omega$ is the center frequency of the input field, one obtains

$$
\frac{d \alpha(t)}{d t}=i \omega_{0} \alpha(t)-\frac{1}{\tau} \alpha(t)+\gamma_{0} s_{\text {in }}(t),
$$

which is the classical equation assumed by Tsakmakidis et al. (Eq. (3) in Ref. [9]). They defined the time-bandwidth product (TBP) as $\tau \gamma_{0}$ and claimed that their proposed device could make $\tau \gamma_{0}$ arbitrarily large.

Assuming the standard bosonic commutation relations $\left[a(0), a^{\dagger}(0)\right]=1$ and $\left[A_{0}(t), A_{0}^{\dagger}\left(t^{\prime}\right)\right]=\delta\left(t-t^{\prime}\right)$, it can be shown that

$$
\left[a(t), a^{\dagger}(t)\right]=e^{-2 t / \tau}+\frac{\tau \gamma_{0}}{2}\left(1-e^{-2 t / \tau}\right) .
$$

Since the Heisenberg-picture operator $a(t)$ in a closed system evolves as $a(t)=U^{+}(t) a(0) U(t)$, where $U(t)$ is a unitary operator, the commutator

$$
\left[a(t), a^{\dagger}(t)\right]=1
$$

must be preserved at all times. The commutator must hold even for open quantum systems, as it is well known that any open quantum system can be modeled as part of a closed system [15]. The commutator preservation forces the TBP to be [10-12]

$$
\tau \gamma_{0}=2
$$

In other words, the decay of the commutator at a rate of $2 / \tau$ must be compensated exactly by coupling to a quantum field at a rate of $\gamma_{0}=2 / \tau$. This is a quantum version of the fluctuationdissipation relation.

Coupling the resonator to more fields via passive linear optics can only make the total dissipation rate $2 / \tau$ larger and the TBP smaller. A general model is

$$
\frac{d a(t)}{d t}=-i \omega_{0} a(t)-\frac{1}{\tau} a(t)+\sum_{j} \sqrt{\gamma_{j}} A_{j}(t),
$$

where $\left\{A_{j}(t) ; j=0,1, \ldots\right\}$ are the annihilation operators of the input fields, obeying $\left[A_{j}(t), A_{k}^{\dagger}\left(t^{\prime}\right)\right]=\delta_{j k} \delta\left(t-t^{\prime}\right)$, and $\left\{\gamma_{j} \geq 0\right\}$ are the coupling rates. To retrieve the classical Eq. (6), the mean field $\left\langle A_{j}(t)\right\rangle$ for $j \neq 0$ should be zero. Equation (8) demands

$$
\tau \sum_{j} \gamma_{j}=2
$$

meaning that

$$
\tau \gamma_{0}=2-\tau \sum_{j \neq 0} \gamma_{j} \leq 2
$$

$\tau \gamma_{0}>2$ implies that there is more quantum fluctuation from an input than dissipation. To keep the system linear and the classical correspondence intact, any modification of Eq. (10) should be additive terms with zero mean, and to make $\tau \gamma_{0}>2$, the net commutator of these additive terms should be negative to preserve Eq. (8). These requirements can be satisfied by introducing a "Bogoliubov" coupling of $a(t)$ to the creation operators $\left\{A_{k}^{\dagger}(t) ; k=1,2, \ldots\right\}$ (excluding $A_{0}^{\dagger}(t)$, which has nonzero mean), as depicted in Fig. 1 and described by

$$
\begin{aligned}
\frac{d a(t)}{d t}= & -i \omega_{0} a(t)-\frac{1}{\tau} a(t)+\sum_{j} \sqrt{\gamma_{j}} A_{j}(t) \\
& +\sum_{k} \sqrt{\kappa_{k}} e^{i \phi_{k}} A_{k}^{\dagger}(t) .
\end{aligned}
$$

where $\left\{\kappa_{k} \geq 0\right\}$ are another set of coupling rates and the phase factors $\left\{\phi_{k}\right\}$ are introduced for generality. The commutator of $a(t)$ becomes

$$
\left[a(t), a^{\dagger}(t)\right]=e^{-2 t / \tau}+\frac{\tau\left(\sum_{j} \gamma_{j}-\sum_{k} \kappa_{k}\right)}{2}\left(1-e^{-2 t / \tau}\right) .
$$

Equation (8) holds when

$$
\tau\left(\sum_{j} \gamma_{j}-\sum_{k} \kappa_{k}\right)=2,
$$

and now $\tau \gamma_{0}=2-\tau \sum_{j \neq 0} \gamma_{j}+\tau \sum_{k} \kappa_{k}$ can exceed 2 and become arbitrarily large. In the limit of $\tau \rightarrow \infty$ and $\sum_{j} \gamma_{j}=$ $\sum_{k} \kappa_{k}$, the total input $\sum_{j} \sqrt{\gamma_{j}} A_{j}(t)+\sum_{k} \sqrt{\kappa_{k}} e^{i \phi_{k}} A_{k}^{\dagger}(t)$ has commuting quadratures, forming a quantum-mechanics-free subsystem $[16,17]$ that does not contribute to the commutator of $a(t)$.

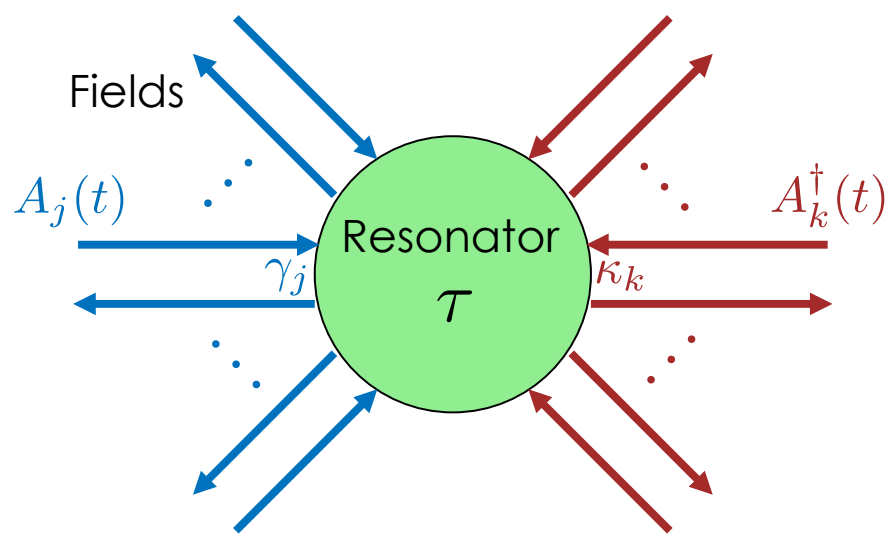

Fig. 1. The general resonator model governed by Eq. (13). Quantum mechanics demands the decay time $\tau$ and the coupling rates $\left\{\gamma_{j}, \kappa_{k}\right\}$ to be related by Eq. (15).

It is well established $[10,11]$ that such a model is equivalent to a resonator with internal amplifiers, in which case $\kappa_{k}$ is the gain coefficient introduced by each amplifier and the net dissipation rate $2 / \tau=\sum_{j} \gamma_{j}-\sum_{k} \kappa_{k}$ is reduced. The presence of $A_{k}^{\dagger}(t)$ terms in Eq. (13), however, must introduce extra noise $[10,11]$, known as amplified spontaneous emission in the context of amplifiers.

When applied to Ref. [9], the quantum models presented here imply one of the following conclusions: 
1. If the physical device proposed by Tsakmakidis et al. obeys the passive model governed by Eq. (10), the TBP cannot exceed 2, meaning that their classical electromagnetic model and simulations are unphysical. A likely explanation is that they failed to account for all dissipation processes in a real device.

2. If the device obeys the general model governed by Eq. (13), it is equivalent to an active resonator and must suffer from extra noise to achieve $\tau \gamma_{0}>2$. There is little practical need for the device when active resonators, an established technology, can do the same job [18]. In fact, active resonators can offer more capabilities via quantum engineering $[5-8,15-17,19-22]$ if the fields are accessible.

3. If the device can achieve $\tau \gamma_{0}>2$ without the extra noise, new physics beyond the textbook models here would be required to explain it. That would be a groundbreaking discovery in quantum optics. A non-Markovian model might offer such a possibility, although the classical correspondence with the Markovian Eq. (6) would likely be lost and the definition of the TBP would need to be modified.

In the context of the nonreciprocal surface magnetoplasmons (SMPs) considered by Tsakmakidis et al., one possible source of extra dissipation is coupling to modes in a neglected third dimension, as explained by Fig. 2 . Their two-dimensional device model and simulations forbid any mode that has a nonzero wavevector component in that dimension and would therefore miss any coupling of the SMPs to those modes in reality. Such turning at a boundary is the more usual behavior of nonreciprocal waves [1-4]. There are two ways of modeling this extra coupling: it can be modeled as extra dissipation for the original resonator mode, in which case $\tau$ will decrease, or the leaked waves can be modeled as part of a redefined resonator supermode, in which case $\gamma$ will decrease, since the rate at which the input can fill the spatially bigger supermode will decrease. Either way, the TBP should decrease from their simulated value.

Thermodynamics can provide another argument that extra dissipation or noise must exist. The model in Ref. [9] relies crucially on the assumption that only a forward-propagating SMP mode exists and the back-propagating mode is forbidden, resulting in a net power transfer and energy build-up in one direction. Suppose that two baths, initially at the same temperature, are placed on the opposite ends of the system, exchanging energy via the SMPs only. The net one-way power transfer would lead to a temperature difference between the two baths, violating the second law of thermodynamics, as depicted in Fig. 3(a). To restore the second law in a closed system, there must be coupling to a third bath that is neglected in their model, as depicted in Fig. 3(b). Whether this coupling to the third bath introduces extra dissipation - thereby reducing the TBP-or extra noise depends on the nature of the coupling.

It is interesting to note that Lord Rayleigh recognized the apparent conflict between nonreciprocal optics and thermodynamics as early as 1885 [23]. Wien proposed a magneto-optical scheme to achieve one-way energy transfer between two ports and violate the second law [24], but Rayleigh realized that Wien's scheme actually involves more than two ports, and closing those extra ports via mirrors makes the two-port coupling rates identical [25]. Similarly, to model a passive two-port optical isolator that absorbs the back-propagating light, a third port that takes the absorbed light should be included, and the

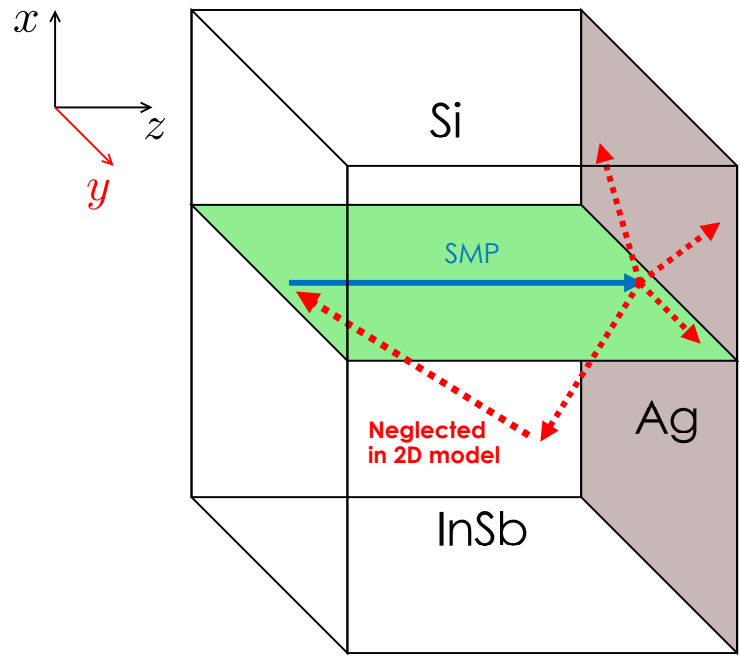

Fig. 2. A three-dimensional view of the silicon-indiumantimonide-silver (Si-InSb-Ag) system assumed in Ref. [9]. Their two-dimensional model with only the $x$ and $z$ dimensions neglect modes that have a nonzero wavevector component in the $y$ dimension $\left(k_{y}\right)$ and would miss any coupling of the SMPs at the Ag mirror to those modes.
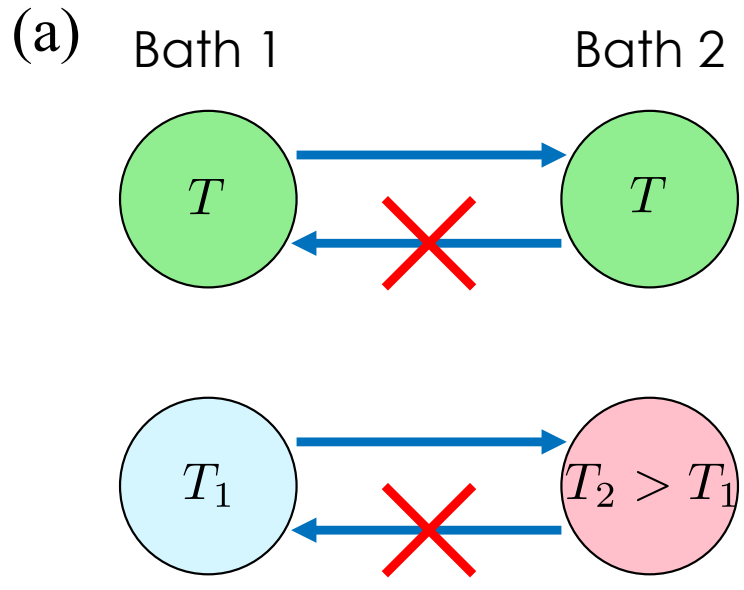

(b)

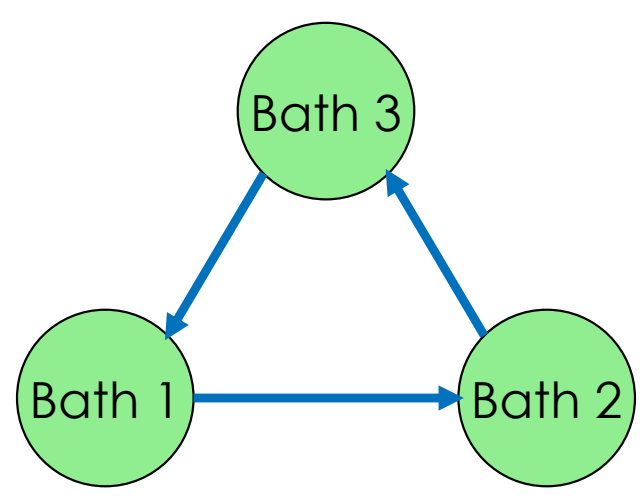

Fig. 3. (a) Two equal-temperature baths undergoing one-way power transfer would develop a temperature difference, violating the second law of thermodynamics. (b) A third bath is needed to restore the second law in a closed nonreciprocal system. 
model becomes equivalent to a three-port circulator. The circulator input-output relations, though nonreciprocal, remain unitary [26] and thus compliant with thermodynamics. Even in the context of SMPs, it has been recognized that thermodynamics mandates nonreciprocal absorption [27] or more than two ports [28] for nonreciprocal reflections to occur. Another interesting example is the chiral edge modes that arise from timereversal-symmetry breaking in quantum Hall systems as well as photonics [1-4]. The modes can propagate in only one direction along the edge of a two-dimensional surface and may sound at odds with thermodynamics, but note that the edge of a finite surface is always a closed curve. Owing to the breaking of time-reversal symmetry, the path that transfers energy from Bath 1 at one point to Bath 2 at another point is not the time reversal of the path that transfers energy from Bath 2 to Bath 1. Both paths do exist on a closed curve, however, and thermodynamics is safe. All these examples show that the modeling of passive nonreciprocal optics requires utmost care, to ensure compliance with not only classical electromagnetism but also thermodynamics.

Regardless of the implementation, the nonreciprocal resonator hypothesized in Ref. [9], with its large input rate and arbitrarily small output rate, is analogous to a black hole that sucks energy in one direction. If there is no other neglected dissipation, extra noise akin to Hawking radiation must be present to uphold the second law. The active-resonator model offers a natural way to extend the analogy to the quantum domain: the type of Bogoliubov coupling used in Eq. (13) also underpins the quantum theory of black-hole radiation [29] and ensures consistency with both quantum mechanics and thermodynamics.

Funding. Singapore Ministry of Education Academic Research Fund Tier 1 Project R-263-000-C06-112.

Interesting discussions with Kosmas Tsakmakidis, who brought Refs. [1-3, 18] to my attention, are gratefully acknowledged.

\section{REFERENCES}

1. S. Raghu and F. D. M. Haldane, Physical Review A 78, 033834 (2008).

2. Z. Wang, Y. D. Chong, J. D. Joannopoulos, and M. Soljačić, Physical Review Letters 100, 013905 (2008).

3. Z. Wang, Y. Chong, J. D. Joannopoulos, and M. Soljačić, Nature 461, 772 (2009).

4. M. Hafezi, S. Mittal, J. Fan, A. Migdall, and J. M. Taylor, Nature Photonics 7, 1001 (2013).

5. J. Koch, A. A. Houck, K. L. Hur, and S. M. Girvin, Physical Review A 82, 043811 (2010).

6. A. Metelmann and A. Clerk, Physical Review X 5, 021025 (2015).

7. K. Fang, J. Luo, A. Metelmann, M. H. Matheny, F. Marquardt, A. A. Clerk, and O. Painter, Nature Physics 13, 465 (2017).

8. L. Tian and Z. Li, Physical Review A 96, 013808 (2017).

9. K. L. Tsakmakidis, L. Shen, S. A. Schulz, X. Zheng, J. Upham, X. Deng, H. Altug, A. F. Vakakis, and R. W. Boyd, Science 356, 1260 (2017).

10. H. A. Haus, Electromagnetic Noise and Quantum Optical Measurements (Springer-Verlag, Berlin, 2000).

11. C. W. Gardiner and P. Zoller, Quantum Noise (Springer-Verlag, Berlin, 2004).

12. L. Mandel and E. Wolf, Optical Coherence and Quantum Optics (Cambridge University Press, Cambridge, 1995).

13. S. L. Braunstein and P. van Loock, Rev. Mod. Phys. 77, 513 (2005).

14. S. Weinberg, The Quantum Theory of Fields. Vol. I. Foundations (Cambridge University Press, Cambridge, 1995).

15. H. M. Wiseman and G. J. Milburn, Quantum Measurement and Control (Cambridge University Press, Cambridge, 2010).

16. M. Tsang and C. M. Caves, Physical Review X 2, 031016 (2012).
17. J. Gough and M. R. James, IEEE Transactions on Automatic Control 54, 2530 (2009).

18. H. Ramezani, J. Schindler, F. M. Ellis, U. Günther, and T. Kottos, Physical Review A 85, 062122 (2012).

19. K. Hammerer, M. Aspelmeyer, E. S. Polzik, and P. Zoller, Physical Review Letters 102, 020501 (2009).

20. M. Tsang and C. M. Caves, Physical Review Letters 105, 123601 (2010).

21. C. B. Møller, R. A. Thomas, G. Vasilakis, E. Zeuthen, Y. Tsaturyan, M. Balabas, K. Jensen, A. Schliesser, K. Hammerer, and E. S. Polzik, Nature 547, 191 (2017).

22. A. Metelmann and A. Clerk, Physical Review Letters 112, 133904 (2014).

23. L. Rayleigh, Philosophical Transactions of the Royal Society of London 176, 343 (1885).

24. W. Wien, "Les lois théoriques du rayonnement," in "Rapports présentés au Congrès International de Physique,", vol. II C. É. Guillaume and L. Poincaré, eds. (1900), vol. II, pp. 23-40.

25. L. Rayleigh, Nature (London) 64, 577 (1901).

26. J. C. Garrison and R. Y. Chiao, Quantum Optics (Oxford University Press, Oxford, 2008).

27. L. Remer, E. Mohler, W. Grill, and B. Lüthi, Physical Review B 30, 3277 (1984).

28. R. L. Stamps, B. L. Johnson, and R. E. Camley, Physical Review B 43, 3626 (1991).

29. S. W. Hawking, Nature 248, 30 (1974). 